\title{
MONOTONE OPERATORS AND NONLINEAR INTEGRAL EQUATIONS OF HAMMERSTEIN TYPE
}

\author{
BY FELIX E. BROWDER AND CHAITAN P. GUPTA
}

Communicated by C. B. Morrey, Jr., May 19, 1969

A nonlinear in tegral equation of Hammerstein type is one of the form

$$
u(x)+\int_{G} K(x, y) f(y, u(y)) d y=0,
$$

where $G$ is a measure space with $\sigma$-finite measure $d y$ and the unknown function $u(x)$ is defined on $G$. In operator-theoretic terms, the problem of determining the solutions of the equation (1) with $u$ lying in a given Banach space $Y$ of functions on $G$ can be put in the form of the nonlinear functional equation

$$
u+A N(u)=0
$$

with the linear and nonlinear mappings $A$ and $N$ given by

$$
A v(x)=\int_{G} K(x, y) v(y) d y, \quad N u(x)=f(x, u(x)) .
$$

In the present note, we establish general results on the existence and uniqueness of solutions of equation (2) for the Banach space $Y=X^{*}$ under appropriate assumptions of weak monotonicity type upon the mappings $A$ and $N$. We note that Hammerstein equations have an extensive literature which includes Hammerstein [11], Iglisch [12], Golomb [10], Dolph [7], Rothe [18], Vainberg [19], [20], and Krasnosel'skiY [16]. The first application of the concept of monotone operator in this problem was made implicitly by Golomb [10] and explicitly by Vainberg [19]. More recent papers applying monotonicity concepts to Hammerstein equations include DolphMinty [8], Kolodner [13], Brézis [3], Kolomy [14], [15], Amann [1], [2], de Figueiredo-Gupta [9] and Vainberg [20].

We employ the following definitions: If $X$ is a real Banach space, $X^{*}$ its conjugate space, we let $(w, u)$ denote the duality pairing between the element $w$ of $X^{*}$ and the element $u$ of $X$. A mapping $A$ of $X$ into $X^{*}$ is said to be monotone if for all $u, v$ in $X$ we have

$$
(A(u)-A(v), u-v) \geqq 0 .
$$

A mapping $N$ of $X^{*}$ into $X$ is said to be hemicontinuous if it is continuous from each line segment of $X^{*}$ to the weak topology of $X$. 
Definition 1. If $A$ is a bounded monotone linear mapping of $X$ into $X^{*}$, then $A$ is said to be angle-bounded with constant $c \geqq 0$ if for all $u, v$ in $X$

$$
|(A(u), v)-(A(v), u)| \leqq 2 c\{(A(u), u)\}^{1 / 2}\{(A(v), v)\}^{1 / 2} .
$$

Definition 2. If $A$ is a bounded linear mapping of $X$ into $X^{*}, A$ is said to be symmetric if for all $u$ and $v$ in $X$,

$$
(A(u), v)=(A(v), u) .
$$

Theorem 1. Let $X$ be a general real Banach space, $X^{*}$ its conjugate space. Let $A$ be a monotone angle-bounded continuous linear mapping of $X$ into $X^{*}$ with constant of angle-boundedness $c \geqq 0$. Let $N$ be a hemicontinuous (possibly nonlinear) mapping of $X^{*}$ into $X$ such that for a given constant $k \geqq 0$

$$
\left(v-v_{1}, N(v)-N\left(v_{1}\right)\right) \geqq-k\left\|v-v_{1}\right\|_{X^{*}}^{2}
$$

for all $v$ and $v_{1}$ in $X^{*}$. Suppose finally that there exists a constant $R$ with $k\left(1+c^{2}\right) R<1$ such that for all $u$ in $X$

$$
(A(u), u) \leqq R\|u\|_{X}^{2} .
$$

Then there exists exactly one solution $w$ in $X^{*}$ of the nonlinear equation

$$
w+A N(w)=0 .
$$

Some special cases of Theorem 1 are the following:

Theorem 2. Let $X$ be a general real Banach space, $X^{*}$ its conjugate space, $A$ a bounded linear mapping of $X$ into $X^{*}$ with $A$ monotone and angle-bounded. Let $N$ be a hemicontinuous (possibly nonlinear) mapping of $X^{*}$ into $X$ which is monotone, that is,

$$
\left(v-v_{1}, N(v)-N\left(v_{1}\right)\right) \geqq 0
$$

for all $v$ and $v_{1}$ in $X^{*}$.

Then there exist exactly one solution w in $X^{*}$ of the equation

$$
w+A N(w)=0 .
$$

The result of Theorem 2 was obtained by Amann [1] under an assumption that $X^{*}$ has a dense continuous linear imbedding in a Hilbert space. As we show below, such assumptions are not needed in the proof of Theorem 1, nor in the proof of Theorem 2 which is a special case of Theorem 1 for $k=0$. Another special case is the following 
THEOREM 3. Let $X$ be a general real Banach space, $X^{*}$ its conjugate space, $A$ a bounded linear mapping of $X$ into $X^{*}$ which is monotone and symmetric. Suppose that $N$ is a hemicontinuous (possibly nonlinear) mapping of $X^{*}$ into $X$ such that for a given $k \geqq 0$ and all $v, v_{1}$ in $X^{*}$,

$$
\left(v-v_{1}, N(v)-N\left(v_{1}\right)\right) \geqq-k\left\|v-v_{1}\right\|_{X^{*} .}^{2}
$$

Suppose that $k\|A\|<1$.

Then the equation $w+A N(w)=0$ has exactly one solution $w$ in $X^{*}$.

We note that when $A$ is symmetric then $A$ is angle-bounded with constant of angle-boundedness $c=0$.

The result of Theorem 3 was obtained by Golomb [10] for $X$ $=L^{2}(G)$ and by Vainberg [20] for $X=L^{p}(G)$, using variational methods. Our method, on the other hand, consists in splitting the linear operator $A$ via a Hilbert space $H$ (Theorem 4) and reducing the equation (6) to an equivalent equation in $H$, which is then solved by using the results of Browder [5] and Minty [17] for monotone operator equations.

We may add that the proof of Theorems 1, 2, 3 and 4 can be adapted in a straightforward manner to the case of a real locallyconvex space.

We turn now to the proof of Theorem 1. An essential tool in that proof is the following auxiliary theorem.

Theorem 4. Let $X$ be a Banach space, $X^{*}$ its conjugate space, $A$ a bounded linear mapping of $X$ into $X^{*}$ which is monotone and anglebounded. Then there exists a Hilbert space $H$, a continuous linear mapping $S$ of $X$ into $H$ with $S^{*}$ injective and a bounded skew-symmetric linear mapping $B$ of $H$ into $H$ such that $A=S^{*}(I+B) S$, and the following two inequalities hold:

(i) $\|B\| \leqq c$, with $c$ the constant of angle-boundedness of $A$.

(ii) $\|S\|^{2} \leqq R$ if and only if for all $u$ in $X,(A(u), u) \leqq R\|u\|_{X}^{2}$.

Proof. We introduce a symmetric bilinear form on $X$ by setting

$$
\left[u, u_{1}\right]=\frac{1}{2}\left\{\left(A(u), u_{1}\right)+\left(A\left(u_{1}\right), u\right)\right\} .
$$

Since $[u, u]=(A(u), u) \geqq 0$ by the monotonicity of $A$, we have the Cauchy-Schwarz inequality

$$
\left|\left[u, u_{1}\right]\right| \leqq\{[u, u]\}^{1 / 2}\left\{\left[u_{1}, u_{1}\right]\right\}^{1 / 2} .
$$

Let $N$ be the subset of those $u$ in $X$ for which $[u, u]=0$. It follows from (7) that $N=\{u \mid u$ in $X,[u, v]=0$ for all $v$ in $X\}$ and hence, $N$ is a closed vector-subspace of $X$. Let $H_{0}$ denote the quotient vector-space 
$X / N$ and endow $H_{0}$ with the inner product $\left[u+N, u_{1}+N\right]=\left[u, u_{1}\right]$. Under this inner product, $H_{0}$ is a pre-Hilbert space. We denote by $H$ the Hilbert space obtained from $H_{0}$ by completing it with respect to this inner product. We let $S$ denote the linear mapping of $X$ into $H$ obtained from the natural projection of $X$ onto $X / N$. Since $A$ is a bounded linear mapping of $X$ into $X^{*}$, there exists a least constant $R \geqq 0$ such that $(A(u), u) \leqq R\|u\|_{X}^{2}$ for all $u$ in $X$. Hence, for all $u$ in $X$ we have

$$
\|S(u)\|_{H}^{2}=[S(u), S(u)]=(A(u), u) \leqq R\|u\|_{X}^{2}
$$

that is, $S$ is a bounded linear mapping of $X$ into $H$ with $\|S\|^{2} \leqq R$. Since the range of $S$ coincides with $H_{0}, S$ has a dense range in $H$. Hence the adjoint mapping $S^{*}$ of $H$ into $X^{*}$ is injective.

Since $A$ is angle-bounded with constant of angle-boundedness $c \geqq 0$, it follows that for all $u, v$ in $X$

$$
|(A(u), v)-(A(v), u)| \leqq 2 c\|S(u)\|_{H}\|S(v)\|_{H} .
$$

Hence the function

$$
h(S(u), S(v))=\frac{1}{2}\{(A(u), v)-(A(v), u)\}
$$

is well defined on $H_{0}$ and is a bounded bilinear form on $H_{0}$. Let $h$ also denote the unique extension of this bounded bilinear form on $H_{0}$ to $H$. It follows that there exists a well-defined and unique bounded linear mapping $B$ of $H$ into $H$ such that for all $u, v$ in $X$

$$
h(S(u), S(v))=[B(S(u)), S(v)] .
$$

Since $h(S(u), S(v))=-h(S(v), S(u))$ for all $u, v$ in $X$, it follows that $B$ is skew-symmetric on $H_{0}$ and hence on all of $H$ by continuity. We note that

$$
|[B(S(u)), S(v)]| \leqq c\|S(u)\|_{H}\|S(v)\|_{H}
$$

for all $u, v$ in $X$ and so $\|B\| \leqq c$.

Finally for all $u, v$ in $X$

$$
\begin{aligned}
(A(u), v) & =[u, v]+h(S(u), S(v))=[S(u), S(v)]+[B(S(u)), S(v)] \\
& =[(I+B) S(u), S(v)]=\left(S^{*}(I+B) S(u), v\right) .
\end{aligned}
$$

Thus $A=S^{*}(I+B) S$. This completes the proof of the theorem.

We also need the following elementary lemma in the proof of Theorem 1.

Lemma. Let $H$ be a given Hilbert space, $B$ a skew-symmetric bounded linear mapping of $H$ into $H$. Then the bounded linear mapping $I+B$ is 
a monotone bijective mapping of $H$ onto $H$. Further, for any $u$ in $H$ we have

$$
\left.\mathrm{L}(I+B)^{-1}(u), u\right] \geqq \frac{1}{1+\|B\|^{2}}\|u\|_{H}^{2} .
$$

Proof of Theorem 1. Suppose $w$ in $X^{*}$ is a solution of the equation (6). By Theorem 4, $A=S^{*}(I+B) S$ and equation (6) becomes

$$
w+S^{*}(I+B) S N(w)=0 .
$$

Since $S^{*}$ is injective, there is a unique $u$ in $H$ such that $w=S^{*}(u)$ and equation (8) becomes

$$
S^{*}(u)+S^{*}(I+B) S N S^{*}(u)=0,
$$

i.e.,

$$
S^{*}\left(u+(I+B) S N S^{*}(u)\right)=0 .
$$

Since $S^{*}$ is injective, equation (10) is equivalent to

$$
u+(I+B) S N S^{*}(u)=0 .
$$

Hence equation (6) has exactly one solution in $X^{*}$ if and only if equation (11) has exactly one solution in $H$. Now, by the lemma, equation (11) is equivalent to the equation

$$
(I+B)^{-1}(u)+S N S^{*}(u)=0 .
$$

Let $T=(I+B)^{-1}+S N S^{*}$. For $u, v$ in $H$,

$$
\begin{aligned}
{[T(u)-T(v), u-v]=} & {\left[(I+B)^{-1}(u-v), u-v\right] } \\
& +\left[S N S^{*}(u)-S N S^{*}(v), u-v\right] .
\end{aligned}
$$

By the lemma and Theorem 4(i)

$$
\left[(I+B)^{-1}(u-v), u-v\right] \geqq \frac{1}{1+\|B\|^{2}}\|u-v\|_{H}^{2} \geqq \frac{1}{1+c^{2}}\|u-v\|_{H}^{2} .
$$

On the other hand,

$$
\begin{aligned}
{\left[S N S^{*}(u)-S N S^{*}(v), u-v\right] } & =\left(S^{*}(u)-S^{*}(v), N S^{*}(u)-N S^{*}(v)\right) \\
& \geqq-k\left\|S^{*}(u)-S^{*}(v)\right\|_{X^{*}}^{2} \geqq-k R\|u-v\|_{H}^{2}
\end{aligned}
$$

by Theorem 4(ii). Combining these inequalities, we see that

$$
[T(u)-T(v), u-v] \geqq\left(1 /\left(1+c^{2}\right)-k R\right)\|u-v\|_{H}^{2} \geqq c_{1}\|u-v\|_{H}^{2}
$$

where $c_{1}=1 /\left(1+c^{2}\right)-k R>0$ since $k\left(1+c^{2}\right) R<1$ by hypothesis. 
Thus $T$ is a monotone hemicontinuous mapping of $H$ into $H$ and $T$ is injective. Moreover, for $u$ in $H$ we have

$$
\begin{aligned}
{[T(u), u] } & =[T(u)-T(0), u-0]+[T(0), u] \\
& \geqq c_{1}\|u\|_{H}^{2}-\|T(0)\|\left\|_{H}\right\| u \|_{H} .
\end{aligned}
$$

Since $[T(u), u] /\|u\|_{H \rightarrow \infty}$ as $\|u\|_{H \rightarrow \infty}, T$ is coercive. It then follows from the results of Browder [5] and Minty [17] that $T$ maps $H$ onto $H$ injectively. Hence (12) has exactly one solution in $H$ and so by our preceding discussion, equation (6) has exactly one solution in $X^{*}$. q.e.d.

\section{BIBLIOGRAPHY}

1. H. Amann, Über die Existenz und iterative Berechnung einer Lösung der Hammersteinschen Gleichung, Aequations Math. 1 (1968), 242-266.

2. - Ein Existenz und Eindeutigkeitssatz für die Hammersteinsche Gleichung in Banachraumen, Math. $Z$. (to appear).

3. H. Brézis, Equations et inequations non-linéaires dans les espaces vectoriels en dualite, Ann. Inst. Fourier (Grenoble) 18 (1968), 115-176.

4. F. E. Browder, The solvability of non-linear functional equations, Duke Math. J. 30 (1963), 557-566.

5. - Nonlinear elliptic boundary value problems, Bull. Amer. Math. Soc. 69 (1963), 862-874.

6. - "Nonlinear operators and nonlinear equations of evolution in Banach spaces" in Non-linear funciional analysis, Proc. Amer. Math. Soc. Sympos. (to appear).

7. C. L. Dolph, Non-linear integral equations of Hammerstein type, Trans. Amer. Math. Soc. 66 (1949), 289-307.

8. C. Dolph and G. J. Minty, On nonlinear integral equations of the Hammerstein type. Nonlinear integral equations, edited by P. M. Anselone, Univ. of Wisconsin Press, Madison, Wis., 1964, pp. 99-154.

9. D. G. de Figueiredo and C. P. Gupta, Solvability of nonlinear integral equations of Hammerstein type (to appear).

10. M. Golomb, On the theory of nonlinear integral equations, integral systems and general functional equations, Math. Z. 39 (1935), 45-75.

11. A. Hammerstein, Nichtlineare Integralgleichungen nebst Anwendungen, Acta Math. 54 (1930), 117-176.

12. R. Iglisch, Existenz und Eindeutigkeit Satze bei nichtlineare Integralgleichungen, Math. Ann. 108 (1933), 161-189.

13. I. I. Kolodner, Equations of Hammerstein type in Hilbert space, J. Math Mech. 13 (1964), 701-750.

14. J. Kolomy, Applications of some existence theorems for the solutions of Hammerstein integral equations, Comment. Math. Univ. Carolinae 7 (1966), 461-478.

15. - The solvability of nonlinear integral equations, Comment. Math. Univ. Carolinae 8 (1967), 273-289.

16. M. A. Krasnosel'skir, Topological methods in the theory of non-linear integral equations, GITTL, Moscow, 1956; English transl., Macmillan, New York, 1964. 
17. G. J. Minty, On a "monotonicity" method for the solution of nonlinear equations in Banach spaces, Proc. Nat. Acad. Sci. U.S.A. 50 (1963), 1038-1041.

18. E. R. Rothe, Weak topology and nonlinear integral equations, Trans. Amer. Math. Soc. 66 (1949), 75-92.

19. M. Vainberg, Variational methods for the study of non-linear operators, GITTL, Moscow, 1956; English transl., Holden-Day, San Francisco, Calif., 1964.

20. - New theorems for nonlinear operators and equations, Moskov. Oblast. Ped. Inst. Ucen Zap. 77 (1959), 131-143. (Russian)

21. - Nonlinear problems for potential and monotone operators, Dokl. Akad. Nauk SSSR 183 (1968), 747-749=Soviet Math. Dokl. 9 (1968), 1427-1430.

University of Chicago, Chicago, Illinois 60637 